\title{
Efficacy and safety of Hydroxychloroquine in treating COVID-19 pneumonia: uncertainty of data and changing treatment protocols
}

\begin{abstract}
The new coronavirus disease which emerged in Wuhan late in 2019 is caused by SARS CoV2, it was named COVID-19 and declared a pandemic by the world health organization (WHO). Human coronavirus normally causes mild disease. The new COVID-19 emerged from bats to human and it's associated with highly infectious disease. The disease clinical features range from an asymptomatic state to mild fever, cough, severe respiratory disease, and multiple organ failures. The disease is confirmed by detecting the virus genome using polymerase chain reaction and antibody detection is used for screening. Radiologic imaging is nonspecific but can help in staging lung involvement. Treatment of patients with COVID-19 is generally supportive, however oxygen and ventilatory support might be needed in some of the cases. Dexamethasone proved to decrease mortality in critically ill patients, but up to date no specific treatment or vaccine is available and many drugs are under clinical trials including ritonavir and remdesivir. Hydroxychloroquine (HCQ) which is an old antimalarial drug, has given hope, but now it's a victim for information uncertainty and contravention of clinical reports. The objective of this article is to review the current reports on hydroxychloroquine efficacy and safety in the treatment of COVID 19 patients.
\end{abstract}

Volume 7 Issue 2 - 2020

\section{Tarig Fadelelmoula}

Department of Respiratory Care,Almaarefa University, Riyadh, Saudi Arabia

Correspondence: Tarig Fadelelmoula, MD, FCCP, Department of Respiratory Care,Almaarefa University, Riyadh, Saudi Arabia, Tel +966542521796, Email ttoo@mcst.edu.sa

Received: August 21, 2020 | Published: September 16, 2020

Keywords: COVID19, hydroxychloroquine, ritonavir, SARS CoV2, Wuhan

\section{Introduction}

The novel coronavirus 19 disease, which emerged in Wuhan of China late in 2019 is caused by the SARS CoV 2 virus which is a member of a coronavirus subfamily named Orthocoronavirinae. Generally, the infection caused by the 2019-nCoV shares many clinical similarities with infection caused by SARS-CoV. ${ }^{1}$ A typical human coronavirus has an incubation period of 2-4 days; it is estimated to be 3-6 days for the 2019-nCoV, and 4-6 days for SARS-CoV. ${ }^{2}$ Symptoms ranged from asymptomatic to severe disease and multiorgan dysfunction. The common symptoms are fever, cough, sore throat, headache, fatigue, headache, myalgia, and breathlessness. ${ }^{3}$ Patients with mild symptoms recover after 1 week but severe cases experience progressive respiratory disease. ${ }^{4}$

Moreover, many patients can be asymptomatic, thus increasing the uncertainty of the diagnostic work-up. Laboratory tests play a pivotal role in the diagnosis and management of COVID-19, the current gold standard being real-time reverse transcription-polymerase chain reaction (rRT-PCR) on respiratory tract specimens. However, the diagnostic accuracy of rRT-PCR depends on many preanalytical and analytical variables. The measurement of specific COVID-19 antibodies (both $\operatorname{IgG} \& \operatorname{IgM}$ ) should serve as an additional, noninvasive tool for disease detection and management. ${ }^{5}$ Viral pneumonia generally shows similar imaging features, some characteristic CT findings may help to differentiate COVID-19 from influenza pneumonia. ${ }^{6}$

\section{Treatment of COVID-19}

Treatment is essentially supportive; the role of antiviral agents is yet to be established. Prevention entails home isolation of suspected cases and those with mild illnesses and strict infection control measures at hospitals that include contact and droplet precautions. ${ }^{7}$ A recent published small clinical trial in France has shown that combination pharmacological treatment with chloroquine (CQ) and hydroxychloroquine (HCQ), an old antimalarial drug, and azithromycin, an antibiotic, led to decreased viral load and an average carrying duration in COVID-19 patients. ${ }^{8}$ Dexamethasone which is an old corticosteroid is now proved to be effective in improving outcomes and decreasing mortality in COVID-19 patients when used in low doses. ${ }^{9}$

\section{Mechanism of action of Hydroxychloroquine}

Studies have demonstrated that HCQ also confers its considerable broad-spectrum antiviral effects via interfering with the fusion process of these viruses by decreasing the $\mathrm{pH}$ and hence conferring antiviral effect. Also, chloroquine alters the glycosylation of the cellular receptors of coronaviruses and interferes with viral entry. ${ }^{10}$ Figure 1 shows phases of the replication of different viruses affected by chloroquine. ${ }^{11}$

\section{Side effects of chloroquine}

Long-term side effects of treatment include retinopathy, vacuolar myopathy, neuropathy, restrictive cardiomyopathy, and cardiac conduction disturbances. ${ }^{12-14}$ however these side effects are negligible in the context of treatment of COVID-19 but may be important if they are used for extended periods of prophylaxis. ${ }^{15}$

\section{Literature review}

A study in France was inspired by a smaller Chinese study showing similar improvements, ${ }^{16}$ these studies have led many healthcare providers globally to utilize hydroxychloroquine/azithromycin combinations to treat COVID-19 pneumonia in critical care settings. ${ }^{17}$ 
In the past 4 Months HCQ use for COVID-19 pneumonia was stopped in many countries in the Middle East following a publication in the lancet: in which the authors concluded that they were unable to confirm the benefit of HCQ or CQ when used alone or with a macrolide on inhospital outcomes for COVID-19 and that each of these drug regimens was associated with decreased in-hospital survival and an increased frequency of ventricular arrhythmias when used for the treatment of COVID-19. ${ }^{18}$ In Saudi Arabia, the MoH removed HCQ from treatment guidelines before introducing it again for treating mild to moderate cases of COVID 19 disease. ${ }^{19}$ In Sudan they introduced HCQ as the main drug for treating confirmed COVID-19 pneumonia patients with mild to moderate disease, and the outcomes were favorable and no adverse events were reported from the different COVD-19 patient treatment centers. ${ }^{20}$ Although some studies raised the possibility of a protective effect for chloroquine and hydroxychloroquine against COVID 19 disease, a report by Gendelman and his colleagues created doubts regarding the protective role of these medications in the battle against SARS-CoV-2 infection. ${ }^{21}$ Interestingly, both CQ and HCQ have been demonstrated to attain high drug levels in many tissues with particular concentration in the lung tissues where concentrations are 200-700 times higher than in the plasma. All these characteristics forecast that chloroquine can report the highest activity in treating early COVID-19 pneumonia or as post-exposure prophylaxis of Health Care Workers accidentally exposed to the virus. ${ }^{22}$ In addition to this, a recent systematic review has clearly stated the existence of preclinical evidence of effectiveness and evidence of safety from longtime clinical use for other indications to justify clinical research on chloroquine in patients with COVID-19.23 A recent review of controlled trials has the use of hydroxychloroquine (HCQ) among outpatients in clinical trials, without high-risk factors for cardiac arrhythmia, is safe with gastrointestinal side effects being the most common side effects and no fatal adverse outcomes were reported (Table 1). ${ }^{24}$ Due to the converse in reports about chloroquine and hydroxychloroquine efficacy and safety some researchers recommended that these medications should be used only for COVID-19 in the case of a carefully designed randomized clinical trial. ${ }^{25}$

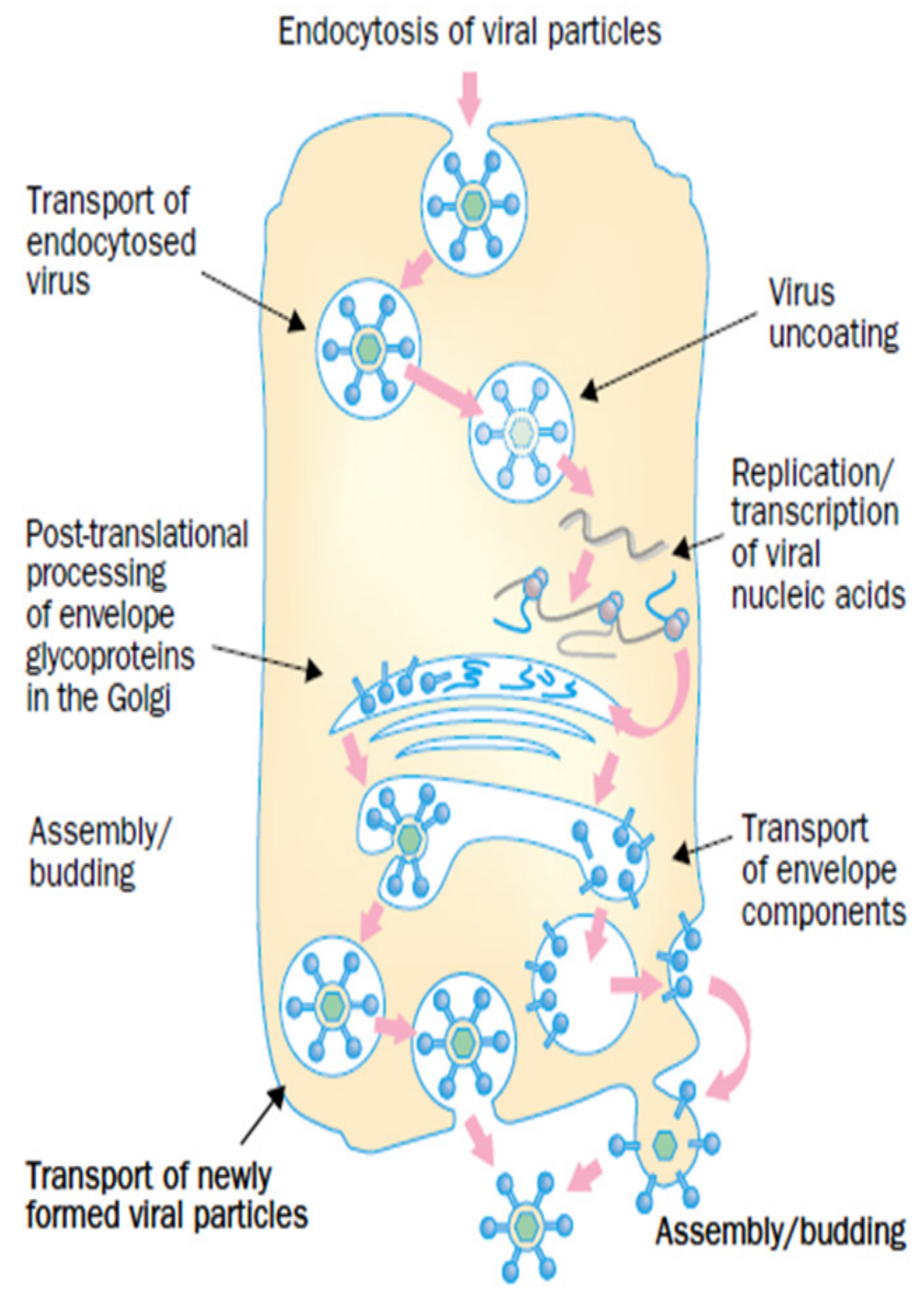

\section{Exocytosis of viral particles}

Figure I Steps of the replication of different viruses affected by Chloroquine (marked by red rectangles). Chloroquine inhibits the replication of different viruses either at the early or late stages of viral replication. 
Table I Combined side effects day I-5 in post exposure prophylaxis and early treatment cohorts

\begin{tabular}{llll}
\hline & Hydroxychtoroquine & Placebo & P-value* \\
\hline Number randomized & 658 & 654 & 0.46 \\
Started study medication & $576(87.5 \%)$ & $563(86.1 \%)$ & $<0.00$ I \\
Any side effect (days I-5). & $231(40.1 \%)$ & $101(17.9 \%)$ &
\end{tabular}

$\begin{array}{ll}\text { Side Effects" } & \\ \text { Nausea or upset stomach } & 146(25.3 \%) \\ \text { Diarrhea, abdominal pain, vomiting } & 131(22.7 \%) \\ \text { Irritability, dizziness, vertigo } & 39(6.8 \%) \\ \text { Tinnitus } & 16(2.8 \%) \\ \text { Headache } & 15(2.6 \%) \\ \text { Visual changes } & 7(1.2 \%) \\ \text { Skin reaction } & 10(1.7 \%) \\ \text { Taste change or dry mouth } & 3(0.5 \%) \\ \text { Allergic reaction } & 6(1.0 \%) \\ \text { Hot flashes, night sweats or palpitations } & 2(0.3 \%) \\ \text { Fatigue } & 1(0.2 \%) \\ \text { Panic } & 0(0.0 \%) \\ \text { Other } & 1(0.2 \%)\end{array}$

*P values calculated via Fisher's Exact test. **Of those who started study medication

\section{Conclusion}

Hydroxychloroquine is an effective, safe, and affordable treatment against COVD19 pneumonia, with increasing reports supporting its use in patients with mild to moderate diseases, however it is use is restricted to clinical trials and it is use outside clinical trials is based on risks and benefits, and a clear beneficial effect remains to be proved.

\section{Acknowledgments}

The author is thankful to Al-Maarefa University, Riyadh, Kingdom of Saudi Arabia, for providing all necessary facilities to carry out and publish this work.

\section{Conflicts of interest}

The author declares there is no conflict of interest.

\section{Funding}

None.

\section{References}

1. Cui J, Li F, Shi L. Origin and evolution of pathogenic coronaviruses. Nat Rev Microbiol. 2019;17:181-192.

2. Huang C, Wang Y, Li X. et al. Clinical features of patients infected with 2019 novel coronavirus in Wuhan, China. Lancet. 2020.

3. Chen N, Zhou M, Dong X, et al. Epidemiological and clinical characteristics of 99 cases of 2019 novel coronavirus pneumonia in Wuhan, China: a descriptive study. Lancet. 2020;395(10223):507-513.
4. Li T, Wei C, Li W, et al. Beijing Union Medical College Hospital on pneumonia of novel coronavirus infection" diagnosis and treatment proposal (V2.0). Med J Peking Union Med Coll Hosp. 2020.

5. Padoan A, Cosma C, Sciacovelli L, et al. Analytical performances of a chemiluminescence immunoassay for SARS-CoV-2 IgM/IgG and antibody kinetics [published online ahead of print, 2020 Apr 16]. Clin Chem Lab Med. 2020;58(7):1081-1088.

6. Wang H, Wei R, Rao G, et al. Characteristic CT findings distinguishing 2019 novel coronavirus disease (COVID-19) from influenza pneumonia [published online ahead of print. Eur Radiol. 2020;30(9):4910-4917.

7. Singhal T. A Review of Coronavirus Disease-2019 (COVID-19). Indian J Pediatr. 2020;87(4):281-286.

8. Gautret P, Lagier JC, Parola P, et al. Hydroxychloroquine and azithromycin as a treatment of COVID-19: results of an open-label non-randomized clinical trial. Int J Antimicrobial Agents. 2020;56(1):105949.

9. Torjesen I. Covid-19: Hydroxychloroquine does not benefit hospitalised patients, UK trial finds. BMJ. 2020;369:m2263.

10. Sahraei Z, Shabani M, Shokouhi S. Aminoquinolines against coronavirus disease 2019 (COVID-19): chloroquine or hydroxychloroquine. Int $J$ Antimicrob Agents. 2020;55(4):105945.

11. Savarino A, Boelaert JR, Cassone A, et al. Effects of chloroquine on viral infections: an old drug against today's diseases?. Lancet Infect Dis. 2003;3(11):722-727.

12. Yusuf IH, Sharma S, Luqmani R, et al. Hydroxychloroquine retinopathy. Eye (Lond). 2017;31(6):828-845.

13. Stein M, Bell MJ, Ang LC. Hydroxychloroquine neuromyotoxicity. $J$ Rheumatol. 2000;27(12):2927-2931. 
14. Chatre C, Roubille F, Vernhet H, et al. Cardiac Complications Attributed to Chloroquine and Hydroxychloroquine: A Systematic Review of the Literature. Drug Saf. 2018;41(10):919-931.

15. Juurlink DN. Safety considerations with chloroquine, hydroxychloroquine and azithromycin in the management of SARS-CoV-2 infection. CMAJ. 2020;192(17):E450-E453.

16. Gao J, Tian Z, Yang X. Breakthrough: chloroquine phosphate has shown apparent efficacy in treatment of COVID-19 associated pneumonia in clinical studies. Biosci Trends. 2020;14(1):72-73.

17. Study to ask: Does antimalarial drug prevent COVID-19?. 2020

18. Mehra MR, Desai SS, Ruschitzka F, et al. Hydroxychloroquine or chloroquine with or without a macrolide for treatment of COVID-19: a multinational registry analysis. Lancet. 2020.

19. Saudi Ministry of Health. Coronavirus Diseases 19 (COVID-19) guidelines. 2020; version 1.8.

20. Sudan Federal Ministry of Health. Coronavirus Diseases 19 (COVID-19) guidelines. 2020 .
21. Gendelman O, Amital H, Bragazzi NL, et al. Continuous hydroxychloroquine or colchicine therapy does not prevent infection with SARS-CoV-2: Insights from a large healthcare database analysis. Autoimmun Rev. 2020;19(7):102566.

22. Esposito S, Noviello S, Pagliano P. Update on treatment of COVID-19: ongoing studies between promising and disappointing results. Infez Med. 2020;28(2):198-211.

23. Cortegiani A, Ingoglia $G$, Ippolito $M$, et al. A systematic review on the efficacy and safety of chloroquine for the treatment of COVID-19. J Crit Care. 2020;57:279-283.

24. Lofgren SMM, Nicol MR, Bangdiwala AS, et al. Safety of Hydroxychloroquine among Outpatient Clinical Trial Participants for COVID-19. Preprint. medRxiv. 2020; 2020.07.16.20155531.

25. Meyerowitz EA, Vannier AGL, Friesen MGN, et al. Rethinking the role of hydroxychloroquine in the treatment of COVID-19. FASEB J. 2020;34(5):6027-6037. 